\title{
Integral inequalities with 'maxima' and their applications to Hadamard type fractional differential equations
}

\author{
Phollakrit Thiramanus ${ }^{1}$, Jessada Tariboon ${ }^{1 *}$ and Sotiris K Ntouyas ${ }^{2}$
}

"Correspondence:

jessadat@kmutnb.ac.th

${ }^{1}$ Nonlinear Dynamic Analysis

Research Center, Department of

Mathematics, Faculty of Applied

Science, King Mongkut's University

of Technology North Bangkok, Bangkok, 10800, Thailand

Full list of author information is available at the end of the article

\begin{abstract}
In this paper, some new integral inequalities with 'maxima' are established involving Hadamard integral. Applications to Hadamard fractional differential equations with 'maxima' are also presented.

MSC: 26A33; 26D10; 26D15

Keywords: integral inequality; differential equations with 'maxima'; fractional differential equations
\end{abstract}

\section{Introduction}

It is well known that integral inequalities play a dominant role in the study of quantitative properties of solutions of differential and integral equations [1-5]. Fractional inequalities are important in studying the existence, uniqueness, and other properties of fractional differential equations. Recently many authors have studied integral inequalities on fractional calculus using Riemann-Liouville and Caputo derivatives; see [6-9] and the references therein. In $[10,11]$, the authors established some weakly singular integral inequalities of Gronwall-Bellman type and also applied them in the qualitative analysis of solutions to certain fractional differential equations of the Caputo type.

Another kind of fractional derivative that appears in the literature is the fractional derivative due to Hadamard, introduced in 1892 [12], which differs from the RiemannLiouville and Caputo derivatives in the sense that the kernel of the integral contains a logarithmic function of an arbitrary exponent. Details and properties of Hadamard fractional derivative and integral can be found in [13-18]. Recently in the literature there appeared some results on fractional integral inequalities using the Hadamard fractional integral; see [19-22].

Let us recall here the definitions of Hadamard's fractional integral and derivative [23].

Definition 1.1 The Hadamard fractional integral of order $\alpha \in \mathbb{R}^{+}$of a function $f(t)$, for all $t>0$, is defined as

$$
H^{\alpha} f(t)=\frac{1}{\Gamma(\alpha)} \int_{0^{+}}^{t}\left(\log \frac{t}{s}\right)^{\alpha-1} f(s) \frac{d s}{s}
$$

where $\Gamma$ is the standard gamma function defined by $\Gamma(\alpha)=\int_{0}^{\infty} e^{-s} s^{\alpha-1} d s$, provided the integral exists, where $\log (\cdot)=\log _{e}(\cdot)$.

\section{囪 Springer}

O2014 Thiramanus et al.; licensee Springer. This is an Open Access article distributed under the terms of the Creative Commons Attribution License (http://creativecommons.org/licenses/by/2.0), which permits unrestricted use, distribution, and reproduction in any medium, provided the original work is properly cited. 
Definition 1.2 The Hadamard fractional derivative of order $\alpha \in[n-1, n), n \in \mathbb{Z}^{+}$, of a function $f(t)$ is given by

$$
{ }_{\mathrm{H}} D^{\alpha} f(t)=\frac{1}{\Gamma(n-\alpha)}\left(t \frac{d}{d t}\right)^{n} \int_{0^{+}}^{t}\left(\log \frac{t}{s}\right)^{n-\alpha-1} f(s) \frac{d s}{s} .
$$

Differential equations with 'maxima' are a special type of differential equations that contain the maximum of the unknown function over a previous interval. Several integral inequalities have been established in the case when the maxima of the unknown scalar function are involved in the integral; see $[24,25]$ and references cited therein.

Recently in [26] some new types of integral inequalities on time scales with 'maxima' have been established, which can be used as a handy tool in the investigation of making estimates for bounds of solutions of dynamic equations on time scales with 'maxima'. In this paper we establish some new integral inequalities with 'maxima' involving Hadamard's integral. The significance of our work lies in the fact that 'maxima' are taken on intervals $[\beta t, t]$ which have non-constant lengths, where $0<\beta<1$. Most papers take the 'maxima' on $[t-h, t]$, where $h>0$ is a given constant.

The paper is organized as follows: in Section 2 we recall some results from [26] in the special case $\mathbb{T}=\mathbb{R}$, used to prove our main results, which are presented in Section 3. In Section 4 we give applications of our results for a Hadamard fractional differential equation with 'maxima'.

\section{Preliminaries}

For convenience we let $t_{0}>0$ throughout. The following results in Lemmas 2.1 and 2.2 are obtained by reducing the time scale $\mathbb{T}=\mathbb{R}, f(t)=g(t) \equiv 1$, and $a(t)=b(t) \equiv 0$ for all $t \in\left(t_{0}, T\right)$ in Theorems 3.3 and 3.2 ([26], p.8 and p.6), respectively.

Lemma 2.1 ([26]) Let the following conditions be satisfied:

$\left(\mathrm{H}_{1}\right)$ The functions $p$ and $q \in C\left(\left(t_{0}, T\right), \mathbb{R}_{+}\right)$.

$\left(\mathrm{H}_{2}\right)$ The function $\phi \in C\left(\left[\beta t_{0}, T\right), \mathbb{R}_{+}\right)$with $\max _{s \in\left[\beta t_{0}, t_{0}\right]} \phi(s)>0$, where $0<\beta<1$.

$\left(\mathrm{H}_{3}\right)$ The function $u \in C\left(\left[\beta t_{0}, T\right), \mathbb{R}_{+}\right)$and satisfies the inequalities

$$
\begin{aligned}
& u(t) \leq \phi(t)+\int_{t_{0}}^{t}\left\{p(s) u(s)+q(s) \max _{\xi \in[\beta s, s]} u(\xi)\right\} d s, \quad t \in\left(t_{0}, T\right), \\
& u(t) \leq \phi(t), \quad t \in\left[\beta t_{0}, t_{0}\right] .
\end{aligned}
$$

Then

$$
u(t) \leq \phi(t)+h(t) \exp \left(\int_{t_{0}}^{t}\{p(s)+q(s)\} d s\right), \quad t \in\left(t_{0}, T\right),
$$

holds, where

$$
h(t)=\max _{s \in\left[\beta t_{0}, t_{0}\right]} \phi(s)+\int_{t_{0}}^{t}\left\{p(s) \phi(s)+q(s) \max _{\xi \in[\beta s, s]} \phi(\xi)\right\} d s, \quad t \in\left(t_{0}, T\right) .
$$

By splitting the initial function $\phi$ into two functions, we deduce the following corollary. 
Corollary 2.1 Let the following conditions be satisfied:

$\left(\mathrm{H}_{4}\right)$ The functions $p, q$, and $v \in C\left(\left(t_{0}, T\right), \mathbb{R}_{+}\right)$.

$\left(\mathrm{H}_{5}\right)$ The function $w \in C\left(\left[\beta t_{0}, t_{0}\right], \mathbb{R}_{+}\right)$with $\max _{s \in\left[\beta t_{0}, t_{0}\right]} w(s)>0$ and $w\left(t_{0}\right)=v\left(t_{0}\right)$, where $0<\beta<1$.

$\left(\mathrm{H}_{6}\right)$ The function $u \in C\left(\left[\beta t_{0}, T\right), \mathbb{R}_{+}\right)$and satisfies the inequalities

$$
\begin{aligned}
& u(t) \leq v(t)+\int_{t_{0}}^{t}\left\{p(s) u(s)+q(s) \max _{\xi \in[\beta s, s]} u(\xi)\right\} d s, \quad t \in\left(t_{0}, T\right), \\
& u(t) \leq w(t), \quad t \in\left[\beta t_{0}, t_{0}\right] .
\end{aligned}
$$

Then

$$
u(t) \leq v(t)+h(t) \exp \left(\int_{t_{0}}^{t}\{p(s)+q(s)\} d s\right), \quad t \in\left(t_{0}, T\right)
$$

holds, where

$$
h(t)=\max _{s \in\left[\beta t_{0}, t_{0}\right]} w(s)+\int_{t_{0}}^{t}\left\{p(s) v(s)+q(s) \max _{\xi \in[\beta s, s]} m(\xi)\right\} d s, \quad t \in\left(t_{0}, T\right),
$$

with

$$
m(t)= \begin{cases}v(t), & t \in\left(t_{0}, T\right), \\ w(t), & t \in\left[\beta t_{0}, t_{0}\right] .\end{cases}
$$

Lemma 2.2 ([26]) Let the condition $\left(\mathrm{H}_{1}\right)$ of Lemma 2.1 is satisfied. In addition, assume that:

$\left(\mathrm{H}_{7}\right)$ The function $k \in C\left(\left(t_{0}, T\right),(0, \infty)\right)$ is nondecreasing.

$\left(\mathrm{H}_{8}\right)$ The function $\phi \in C\left(\left[\beta t_{0}, t_{0}\right), \mathbb{R}_{+}\right)$, where $0<\beta<1$.

$\left(\mathrm{H}_{9}\right)$ The function $u \in C\left(\left[\beta t_{0}, T\right), \mathbb{R}_{+}\right)$and satisfies the inequalities

$$
\begin{aligned}
& u(t) \leq k(t)+\int_{t_{0}}^{t}\left\{p(s) u(s)+q(s) \max _{\xi \in[\beta s, s]} u(\xi)\right\} d s, \quad t \in\left(t_{0}, T\right), \\
& u(t) \leq \phi(t), \quad t \in\left[\beta t_{0}, t_{0}\right] .
\end{aligned}
$$

Then

$$
u(t) \leq N k(t) \exp \left(\int_{t_{0}}^{t}\{p(s)+q(s)\} d s\right), \quad t \in\left(t_{0}, T\right),
$$

holds, where

$$
N=\max \left\{1, \frac{\max _{s \in\left[\beta t_{0}, t_{0}\right]} \phi(s)}{k\left(t_{0}\right)}\right\} .
$$

The following lemma is a consequence of Jensen's inequality, which can be found in [27]. 
Lemma 2.3 ([27]) Let $n \in N$, and let $x_{1}, \ldots, x_{n}$ be non-negative real numbers. Then for $\sigma>1$,

$$
\left(\sum_{i=1}^{n} x_{i}\right)^{\sigma} \leq n^{\sigma-1} \sum_{i=1}^{n} x_{i}^{\sigma}
$$

\section{Main results}

Theorem 3.1 Suppose that the following conditions are satisfied:

$\left(\mathrm{A}_{1}\right)$ The functions $p$ and $r \in C\left(\left(t_{0}, T\right), \mathbb{R}_{+}\right)$.

$\left(\mathrm{A}_{2}\right)$ The function $\phi \in C\left(\left[\beta t_{0}, t_{0}\right], \mathbb{R}_{+}\right)$with $\max _{s \in\left[\beta t_{0}, t_{0}\right]} \phi(s)>0$, where $0<\beta<1$.

$\left(\mathrm{A}_{3}\right)$ The function $u \in C\left(\left[\beta t_{0}, T\right), \mathbb{R}_{+}\right)$with

$$
\begin{aligned}
& u(t) \leq r(t)+\int_{t_{0}}^{t}\left(\log \frac{t}{s}\right)^{\alpha-1} p(s) \max _{\xi \in[\beta s, s]} u(\xi) \frac{d s}{s}, \quad t \in\left(t_{0}, T\right), \\
& u(t) \leq \phi(t), \quad t \in\left[\beta t_{0}, t_{0}\right],
\end{aligned}
$$

where $\alpha>0$.

Then the following assertions hold:

(i) Suppose $\alpha>\frac{1}{2}$, then

$$
u(t) \leq t\left[c_{1} r^{2}(t)+h_{1}(t) \exp \left(\frac{2 \Gamma(2 \alpha-1)}{t} \int_{t_{0}}^{t} p^{2}(s) d s\right)\right]^{\frac{1}{2}}, \quad t \in\left(t_{0}, T\right),
$$

where

$$
c_{1}=\max \left\{2 t_{0}^{-2},\left(\beta t_{0}\right)^{-2}\right\}
$$

and

$$
\begin{aligned}
h_{1}(t)= & c_{1} \max _{s \in\left[\beta t_{0}, t_{0}\right]} \phi^{2}(s)+\frac{2 c_{1} \Gamma(2 \alpha-1)}{t} \\
& \times \int_{t_{0}}^{t} p^{2}(s) \max _{\xi \in[\beta s, s]} m_{1}^{2}(\xi) d s, \quad t \in\left(t_{0}, T\right),
\end{aligned}
$$

with

$$
m_{1}(t)= \begin{cases}r(t), & t \in\left(t_{0}, T\right) \\ \phi(t), & t \in\left[\beta t_{0}, t_{0}\right]\end{cases}
$$

In addition, if $r \in C\left(\left(t_{0}, T\right),(0, \infty)\right)$ is a nondecreasing function, then

$$
u(t) \leq \sqrt{c_{1} N_{1}} \operatorname{tr}(t) \exp \left(\frac{\Gamma(2 \alpha-1)}{t} \int_{t_{0}}^{t} p^{2}(s) d s\right), \quad t \in\left(t_{0}, T\right),
$$

where

$$
N_{1}=\max \left\{1, \frac{\max _{s \in\left[\beta t_{0}, t_{0}\right]} \phi^{2}(s)}{r^{2}\left(t_{0}\right)}\right\} .
$$


(ii) Suppose $0<\alpha \leq \frac{1}{2}$, then

$$
u(t) \leq t\left[c_{2} r^{b}(t)+h_{2}(t) \exp \left(\frac{\left(2 \Gamma\left(\alpha^{2}\right)\right)^{\frac{1}{\alpha}}}{t} \int_{t_{0}}^{t} p^{b}(s) d s\right)\right]^{\frac{1}{b}}, \quad t \in\left(t_{0}, T\right)
$$

where $b=1+\frac{1}{\alpha}$,

$$
c_{2}=\max \left\{2^{\frac{1}{\alpha}} t_{0}^{-b},\left(\beta t_{0}\right)^{-b}\right\}
$$

and

$$
\begin{aligned}
h_{2}(t)= & c_{2} \max _{s \in\left[\beta t_{0}, t_{0}\right]} \phi^{b}(s)+\frac{c_{2}\left(2 \Gamma\left(\alpha^{2}\right)\right)^{\frac{1}{\alpha}}}{t} \\
& \times \int_{t_{0}}^{t} p^{b}(s) \max _{\xi \in[\beta s, s]} m_{1}^{b}(\xi) d s, \quad t \in\left(t_{0}, T\right) .
\end{aligned}
$$

Moreover, if $r \in C\left(\left(t_{0}, T\right),(0, \infty)\right)$ is a nondecreasing function, then

$$
u(t) \leq\left(c_{2} N_{2}\right)^{\frac{1}{b}} \operatorname{tr}(t) \exp \left(\frac{\left(2 \Gamma\left(\alpha^{2}\right)\right)^{\frac{1}{\alpha}}}{b t} \int_{t_{0}}^{t} p^{b}(s) d s\right), \quad t \in\left(t_{0}, T\right)
$$

where

$$
N_{2}=\max \left\{1, \frac{\max _{s \in\left[\beta t_{0}, t_{0}\right]} \phi^{b}(s)}{r^{b}\left(t_{0}\right)}\right\}
$$

Proof (i) $\alpha>\frac{1}{2}$. For $t \in\left(t_{0}, T\right)$, by using the Cauchy-Schwarz inequality in (3.1), we get

$$
\begin{aligned}
u(t) \leq & r(t)+\left\{\int_{t_{0}}^{t}\left(\log \frac{t}{s}\right)^{2 \alpha-2} d s\right\}^{\frac{1}{2}} \\
& \times\left\{\int_{t_{0}}^{t} p^{2}(s)\left(\max _{\xi \in[\beta s, s]} u(\xi)\right)^{2} \frac{d s}{s^{2}}\right\}^{\frac{1}{2}} .
\end{aligned}
$$

It is easy to observe that

$$
\int_{t_{0}}^{t}\left(\log \frac{t}{s}\right)^{2 \alpha-2} d s=t \int_{0}^{\log \frac{t}{t_{0}}} \tau^{2 \alpha-2} e^{-\tau} d \tau<\Gamma(2 \alpha-1) t
$$

Substituting (3.15) in (3.14), we obtain

$$
u(t) \leq r(t)+(\Gamma(2 \alpha-1) t)^{\frac{1}{2}}\left\{\int_{t_{0}}^{t} p^{2}(s)\left(\max _{\xi \in[\beta s, s]} u(\xi)\right)^{2} \frac{d s}{s^{2}}\right\}^{\frac{1}{2}}
$$

Applying Lemma 2.3 with $n=2, \sigma=2$, we get the estimate

$$
u^{2}(t) \leq 2 r^{2}(t)+2 \Gamma(2 \alpha-1) t \int_{t_{0}}^{t} p^{2}(s)\left(\max _{\xi \in[\beta s, s]} u(\xi)\right)^{2} \frac{d s}{s^{2}}, \quad t \in\left(t_{0}, T\right) .
$$


Setting $v(t)=t^{-2} u^{2}(t)$, we have, for $t \in\left(t_{0}, T\right)$,

$$
\begin{aligned}
v(t) & \leq 2 t^{-2} r^{2}(t)+\frac{2 \Gamma(2 \alpha-1)}{t} \int_{t_{0}}^{t} p^{2}(s)\left(\max _{\xi \in[\beta s, s]} u(\xi)\right)^{2} \frac{d s}{s^{2}} \\
& \leq 2 t_{0}^{-2} r^{2}(t)+\frac{2 \Gamma(2 \alpha-1)}{t} \int_{t_{0}}^{t} p^{2}(s) \max _{\xi \in[\beta s, s]}\left(\xi^{-2} u^{2}(\xi)\right) d s \\
& \leq c_{1} r^{2}(t)+\frac{2 \Gamma(2 \alpha-1)}{t} \int_{t_{0}}^{t} p^{2}(s) \max _{\xi \in[\beta s, s]} v(\xi) d s,
\end{aligned}
$$

and for $t \in\left[\beta t_{0}, t_{0}\right]$,

$$
v(t) \leq t^{-2} \phi^{2}(t) \leq\left(\beta t_{0}\right)^{-2} \phi^{2}(t) \leq c_{1} \phi^{2}(t) .
$$

A suitable application of Corollary 2.1 for (3.16) and (3.17) leads to

$$
v(t) \leq c_{1} r^{2}(t)+h_{1}(t) \exp \left(\frac{2 \Gamma(2 \alpha-1)}{t} \int_{t_{0}}^{t} p^{2}(s) d s\right), \quad t \in\left(t_{0}, T\right),
$$

where $c_{1}$ and $h_{1}$ are defined by (3.4) and (3.5), respectively. Therefore, we obtain the desired bound in (3.3).

Now, if $r \in C\left(\left(t_{0}, T\right),(0, \infty)\right)$ is a nondecreasing function, then, by Lemma 2.2 with (3.16) and (3.17), it follows that

$$
v(t) \leq c_{1} N_{1} r^{2}(t) \exp \left(\frac{2 \Gamma(2 \alpha-1)}{t} \int_{t_{0}}^{t} p^{2}(s) d s\right), \quad t \in\left(t_{0}, T\right),
$$

where $N_{1}$ is defined by (3.8). Thus, we get the inequality in (3.7). This completes the proof of the first part.

(ii) $0<\alpha \leq \frac{1}{2}$. Let $a=1+\alpha$ and $b=1+\frac{1}{\alpha}$. It is obvious that $\frac{1}{a}+\frac{1}{b}=1$. Using the Hölder inequality in (3.1), for $t \in\left(t_{0}, T\right)$, we obtain

$$
u(t) \leq r(t)+\left\{\int_{t_{0}}^{t}\left(\log \frac{t}{s}\right)^{a(\alpha-1)} d s\right\}^{\frac{1}{a}}\left\{\int_{t_{0}}^{t} p^{b}(s)\left(\max _{\xi \in[\beta s, s]} u(\xi)\right)^{b} \frac{d s}{s^{b}}\right\}^{\frac{1}{b}} .
$$

For the first integral in (3.18), repeating the process to get (3.15), we have

$$
\int_{t_{0}}^{t}\left(\log \frac{t}{s}\right)^{a(\alpha-1)} d s<\Gamma(1-a(1-\alpha)) t
$$

Obviously, $1-a(1-\alpha)=\alpha^{2}>0$ and $\Gamma(1-a(1-\alpha)) \in \mathbb{R}$. Substituting (3.19) in (3.18), we get

$$
u(t) \leq r(t)+\left(\Gamma\left(\alpha^{2}\right) t\right)^{\frac{1}{a}}\left\{\int_{t_{0}}^{t} p^{b}(s)\left(\max _{\xi \in[\beta, s]} u(\xi)\right)^{b} \frac{d s}{s^{b}}\right\}^{\frac{1}{b}} .
$$

Applying Lemma 2.3 with $n=2, \sigma=b$, we get the following estimate:

$$
\begin{aligned}
u^{b}(t) & \leq 2^{b-1} r^{b}(t)+2^{b-1}\left(\Gamma\left(\alpha^{2}\right) t\right)^{\frac{b}{a}} \int_{t_{0}}^{t} p^{b}(s)\left(\max _{\xi \in[\beta s, s]} u(\xi)\right)^{b} \frac{d s}{s^{b}} \\
& =2^{\frac{1}{\alpha}} r^{b}(t)+\left(2 \Gamma\left(\alpha^{2}\right) t\right)^{\frac{1}{\alpha}} \int_{t_{0}}^{t} p^{b}(s)\left(\max _{\xi \in[\beta s, s]} u(\xi)\right)^{b} \frac{d s}{s^{b}}, \quad t \in\left(t_{0}, T\right) .
\end{aligned}
$$


By taking $v(t)=t^{-b} u^{b}(t)$, we have

$$
v(t) \leq c_{2} r^{b}(t)+\frac{\left(2 \Gamma\left(\alpha^{2}\right)\right)^{\frac{1}{\alpha}}}{t} \int_{t_{0}}^{t} p^{b}(s) \max _{\xi \in[\beta s, s]} v(\xi) d s, \quad t \in\left(t_{0}, T\right)
$$

and

$$
v(t) \leq c_{2} \phi^{b}(t), \quad t \in\left[\beta t_{0}, t_{0}\right] .
$$

An application of Corollary 2.1 to (3.20) and (3.21) yields

$$
v(t) \leq c_{2} r^{b}(t)+h_{2}(t) \exp \left(\frac{\left(2 \Gamma\left(\alpha^{2}\right)\right)^{\frac{1}{\alpha}}}{t} \int_{t_{0}}^{t} p^{b}(s) d s\right), \quad t \in\left(t_{0}, T\right),
$$

where $c_{2}$ and $h_{2}$ are defined by (3.10) and (3.11), respectively. Thus, we get the required inequality in (3.9).

Furthermore, if $r \in C\left(\left(t_{0}, T\right),(0, \infty)\right)$ is a nondecreasing function, then, by applying Lemma 2.2 to (3.21) and (3.22), we get

$$
v(t) \leq c_{2} N_{2} r^{b}(t) \exp \left(\frac{\left(2 \Gamma\left(\alpha^{2}\right)\right)^{\frac{1}{\alpha}}}{t} \int_{t_{0}}^{t} p^{b}(s) d s\right), \quad t \in\left(t_{0}, T\right),
$$

where $N_{2}$ is defined by (3.13). Therefore, the desired inequality (3.12) is proved. This completes the proof.

Theorem 3.2 Suppose that the conditions $\left(\mathrm{A}_{1}\right)$ and $\left(\mathrm{A}_{2}\right)$ of Theorem 3.1 are satisfied. In addition we assume that:

$\left(\mathrm{A}_{4}\right)$ The function $q \in C\left(\left(t_{0}, T\right), \mathbb{R}_{+}\right)$.

$\left(\mathrm{A}_{5}\right)$ The function $u \in C\left(\left[\beta t_{0}, T\right), \mathbb{R}_{+}\right)$with

$$
\begin{aligned}
& u(t) \leq r(t)+\int_{t_{0}}^{t}\left(\log \frac{t}{s}\right)^{\alpha-1}\left\{p(s) u(s)+q(s) \max _{\xi \in[\beta s, s]} u(\xi)\right\} \frac{d s}{s}, \quad t \in\left(t_{0}, T\right), \\
& u(t) \leq \phi(t), \quad t \in\left[\beta t_{0}, t_{0}\right],
\end{aligned}
$$

where $\alpha>0$.

Then the following assertions hold:

(a) Suppose $\alpha>\frac{1}{2}$, then

$$
\begin{aligned}
& u(t) \leq t\left\{c_{3} r^{2}(t)+h_{3}(t) \exp \left(\frac{3 \Gamma(2 \alpha-1)}{t} \int_{t_{0}}^{t}\left\{p^{2}(s)+q^{2}(s)\right\} d s\right)\right\}^{\frac{1}{2}}, \\
& t \in\left(t_{0}, T\right)
\end{aligned}
$$

where

$$
c_{3}=\max \left\{3 t_{0}^{-2},\left(\beta t_{0}\right)^{-2}\right\}
$$


Thiramanus et al. Journal of Inequalities and Applications 2014, 2014:398

Page 8 of 15

http://www.journalofinequalitiesandapplications.com/content/2014/1/398

and

$$
\begin{aligned}
h_{3}(t)= & c_{3} \max _{s \in\left[\beta t_{0}, t_{0}\right]} \phi^{2}(s)+\frac{3 c_{3} \Gamma(2 \alpha-1)}{t} \\
& \times \int_{t_{0}}^{t}\left\{p^{2}(s) r^{2}(s)+q^{2}(s) \max _{\xi \in[\beta s, s]} m_{1}^{2}(\xi)\right\} d s, \quad t \in\left(t_{0}, T\right),
\end{aligned}
$$

with $m_{1}$ is defined by (3.6).

Furthermore, if $r \in C\left(\left(t_{0}, T\right),(0, \infty)\right)$ is a nondecreasing function, then

$$
u(t) \leq \sqrt{c_{3} N_{1}} \operatorname{tr}(t) \exp \left(\frac{3 \Gamma(2 \alpha-1)}{2 t} \int_{t_{0}}^{t}\left\{p^{2}(s)+q^{2}(s)\right\} d s\right), \quad t \in\left(t_{0}, T\right)
$$

where $N_{1}$ is defined by (3.8).

(b) Suppose $0<\alpha \leq \frac{1}{2}$, then

$$
\begin{aligned}
& u(t) \leq t\left\{c_{4} r^{b}(t)+h_{4}(t) \exp \left(\frac{\left(3 \Gamma\left(\alpha^{2}\right)\right)^{\frac{1}{\alpha}}}{t} \int_{t_{0}}^{t}\left\{p^{b}(s)+q^{b}(s)\right\} d s\right)\right\}^{\frac{1}{b}} \\
& t \in\left(t_{0}, T\right)
\end{aligned}
$$

where $b=1+\frac{1}{\alpha}$,

$$
c_{4}=\max \left\{3^{\frac{1}{\alpha}} t_{0}^{-b},\left(\beta t_{0}\right)^{-b}\right\}
$$

and

$$
\begin{aligned}
h_{4}(t)= & c_{4} \max _{s \in\left[\beta t_{0}, t_{0}\right]} \phi^{b}(s)+\frac{c_{4}\left(3 \Gamma\left(\alpha^{2}\right)\right)^{\frac{1}{\alpha}}}{t} \\
& \times \int_{t_{0}}^{t}\left\{p^{b}(s) r^{b}(s)+q^{b}(s) \max _{\xi \in[\beta s, s]} m_{1}^{b}(\xi)\right\} d s, \quad t \in\left(t_{0}, T\right) .
\end{aligned}
$$

In addition, if $r \in C\left(\left(t_{0}, T\right),(0, \infty)\right)$ is a nondecreasing function, then

$$
u(t) \leq\left(c_{4} N_{2}\right)^{\frac{1}{b}} \operatorname{tr}(t) \exp \left(\frac{\left(3 \Gamma\left(\alpha^{2}\right)\right)^{\frac{1}{\alpha}}}{b t} \int_{t_{0}}^{t}\left\{p^{b}(s)+q^{b}(s)\right\} d s\right), \quad t \in\left(t_{0}, T\right)
$$

where $\mathrm{N}_{2}$ is defined by (3.13).

Proof (a) $\alpha>\frac{1}{2}$. By using the Cauchy-Schwarz inequality in (3.22), for $t \in\left(t_{0}, T\right)$, we have

$$
\begin{aligned}
u(t) \leq & r(t)+\left\{\int_{t_{0}}^{t}\left(\log \frac{t}{s}\right)^{2 \alpha-2} d s\right\}^{\frac{1}{2}}\left\{\int_{t_{0}}^{t} p^{2}(s) u^{2}(s) \frac{d s}{s^{2}}\right\}^{\frac{1}{2}} \\
& +\left\{\int_{t_{0}}^{t}\left(\log \frac{t}{s}\right)^{2 \alpha-2} d s\right\}^{\frac{1}{2}}\left\{\int_{t_{0}}^{t} q^{2}(s)\left(\max _{\xi \in[\beta s, s]} u(\xi)\right)^{2} \frac{d s}{s^{2}}\right\}^{\frac{1}{2}} \\
\leq & r(t)+(\Gamma(2 \alpha-1) t)^{\frac{1}{2}}\left\{\left(\int_{t_{0}}^{t} p^{2}(s) u^{2}(s) \frac{d s}{s^{2}}\right)^{\frac{1}{2}}\right. \\
& \left.+\left(\int_{t_{0}}^{t} q^{2}(s)\left(\max _{\xi \in[\beta s, s]} u(\xi)\right)^{2} \frac{d s}{s^{2}}\right)^{\frac{1}{2}}\right\} .
\end{aligned}
$$


Thiramanus et al. Journal of Inequalities and Applications 2014, 2014:398

Page 9 of 15

By applying Lemma 2.3 with $n=3, \sigma=2$, we get

$$
\begin{aligned}
u^{2}(t) \leq & 3 r^{2}(t)+3 \Gamma(2 \alpha-1) t\left\{\int_{t_{0}}^{t} p^{2}(s) u^{2}(s) \frac{d s}{s^{2}}\right. \\
& \left.+\int_{t_{0}}^{t} q^{2}(s)\left(\max _{\xi \in[\beta s, s]} u(\xi)\right)^{2} \frac{d s}{s^{2}}\right\}, \quad t \in\left(t_{0}, T\right) .
\end{aligned}
$$

Setting $v(t)=t^{-2} u^{2}(t)$, we obtain

$$
\begin{aligned}
v(t) \leq & c_{3} r^{2}(t)+\frac{3 \Gamma(2 \alpha-1)}{t}\left\{\int_{t_{0}}^{t} p^{2}(s) v(s) d s\right. \\
& \left.+\int_{t_{0}}^{t} q^{2}(s) \max _{\xi \in[\beta s, s]} v(\xi) d s\right\}, \quad t \in\left(t_{0}, T\right)
\end{aligned}
$$

and

$$
v(t) \leq c_{3} \phi^{2}(t), \quad t \in\left[\beta t_{0}, t_{0}\right]
$$

Using Corollary 2.1 for (3.32) and (3.33), it follows that

$$
v(t) \leq c_{3} r^{2}(t)+h_{3}(t) \exp \left(\frac{3 \Gamma(2 \alpha-1)}{t} \int_{t_{0}}^{t}\left\{p^{2}(s)+q^{2}(s)\right\} d s\right), \quad t \in\left(t_{0}, T\right),
$$

where $c_{3}$ and $h_{3}$ are defined by (3.25) and (3.26), respectively. Therefore, we get the desired inequality in (3.24).

As a special case, if $r \in C\left(\left(t_{0}, T\right),(0, \infty)\right)$ is a nondecreasing function, then by applying Lemma 2.2 with (3.32) and (3.33), we have

$$
v(t) \leq c_{3} N_{1} r^{2}(t) \exp \left(\frac{3 \Gamma(2 \alpha-1)}{t} \int_{t_{0}}^{t}\left\{p^{2}(s)+q^{2}(s)\right\} d s\right), \quad t \in\left(t_{0}, T\right),
$$

where $N_{1}$ is defined by (3.8). Thus, we get the required inequality in (3.27). This completes the proof of the first part.

(b) $0<\alpha \leq \frac{1}{2}$. Let $a=1+\alpha$ and $b=1+\frac{1}{\alpha}$. Using the Hölder inequality in (3.22), for $t \in\left(t_{0}, T\right)$, we obtain

$$
\begin{aligned}
u(t) \leq & r(t)+\left\{\int_{t_{0}}^{t}\left(\log \frac{t}{s}\right)^{a(\alpha-1)} d s\right\}^{\frac{1}{a}}\left\{\left(\int_{t_{0}}^{t} p^{b}(s) u^{b}(s) \frac{d s}{s^{b}}\right)^{\frac{1}{b}}\right. \\
& \left.+\left(\int_{t_{0}}^{t} q^{b}(s)\left(\max _{\xi \in[\beta s, s]} u(\xi)\right)^{b} \frac{d s}{s^{b}}\right)^{\frac{1}{b}}\right\} \\
\leq & r(t)+\left(\Gamma\left(\alpha^{2}\right) t\right)^{\frac{1}{a}}\left\{\left(\int_{t_{0}}^{t} p^{b}(s) u^{b}(s) \frac{d s}{s^{b}}\right)^{\frac{1}{b}}\right. \\
& \left.+\left(\int_{t_{0}}^{t} q^{b}(s)\left(\max _{\xi \in[\beta s, s]} u(\xi)\right)^{b} \frac{d s}{s^{b}}\right)^{\frac{1}{b}}\right\} .
\end{aligned}
$$


By applying Lemma 2.3 with $n=3, \sigma=b$, we get

$$
\begin{aligned}
u^{b}(t) \leq & 3^{\frac{1}{\alpha}} r^{b}(t)+\left(3 \Gamma\left(\alpha^{2}\right) t\right)^{\frac{1}{\alpha}}\left\{\int_{t_{0}}^{t} p^{b}(s) u^{b}(s) \frac{d s}{s^{b}}\right. \\
& \left.+\int_{t_{0}}^{t} q^{b}(s)\left(\max _{\xi \in[\beta s, s]} u(\xi)\right)^{b} \frac{d s}{s^{b}}\right\}, \quad t \in\left(t_{0}, T\right) .
\end{aligned}
$$

Taking $v(t)=t^{-b} u^{b}(t)$, it follows that

$$
\begin{aligned}
v(t) \leq & c_{4} r^{b}(t)+\frac{\left(3 \Gamma\left(\alpha^{2}\right)\right)^{\frac{1}{\alpha}}}{t}\left\{\int_{t_{0}}^{t} p^{b}(s) v(s) d s\right. \\
& \left.+\int_{t_{0}}^{t} q^{b}(s) \max _{\xi \in[\beta s, s]} v(\xi) d s\right\}, \quad t \in\left(t_{0}, T\right)
\end{aligned}
$$

and

$$
v(t) \leq c_{4} \phi^{b}(t), \quad t \in\left[\beta t_{0}, t_{0}\right]
$$

Applying Corollary 2.1 for (3.34) and (3.35), we have the following estimate:

$$
v(t) \leq c_{4} r^{b}(t)+h_{4}(t) \exp \left(\frac{\left(3 \Gamma\left(\alpha^{2}\right)\right)^{\frac{1}{\alpha}}}{t} \int_{t_{0}}^{t}\left\{p^{b}(s) d+q^{b}(s)\right\} d s\right), \quad t \in\left(t_{0}, T\right)
$$

where $c_{4}$ and $h_{4}$ are defined by (3.29) and (3.30), respectively. Hence, the result (3.28) is proved.

As a special case, if $r \in C\left(\left(t_{0}, T\right),(0, \infty)\right)$ is a nondecreasing function, then by using Lemma 2.2 with (3.34) and (3.35), we get

$$
v(t) \leq c_{4} N_{2} r^{b}(t) \exp \left(\frac{\left(3 \Gamma\left(\alpha^{2}\right)\right)^{\frac{1}{\alpha}}}{t} \int_{t_{0}}^{t}\left\{p^{b}(s) d+q^{b}(s)\right\} d s\right), \quad t \in\left(t_{0}, T\right),
$$

where $N_{2}$ is defined by (3.13). Thus, the required inequality in (3.31) is proved. This completes the proof.

\section{Applications to Hadamard fractional differential equations with 'maxima'}

In this section, the dependence of solutions on the orders with initial conditions and the bound of solutions for the Hadamard fractional differential equations, are investigated. We consider the following fractional differential equation with 'maxima':

$$
\begin{aligned}
& { }_{\mathrm{H}} D^{\alpha} y(t)=f\left(t, y(t), \max _{s \in[\beta t, t]} y(s)\right), \quad t \in I=\left(t_{0}, T\right), \\
& \left.{ }_{\mathrm{H}} D^{\alpha-k} y(t)\right|_{t=t_{0}^{+}}=\eta_{k}, \quad k=1,2, \ldots, n, n=-[-\alpha],
\end{aligned}
$$

and the initial function

$$
y(t)=\phi(t), \quad t \in\left[\beta t_{0}, t_{0}\right]
$$


where ${ }_{\mathrm{H}} D^{\alpha}$ represents the Hadamard fractional derivative of order $\alpha(\alpha>0), f \in C(I \times$ $\mathbb{R} \times \mathbb{R}, \mathbb{R}), \phi$ is a given continuous function on $\left[\beta t_{0}, t_{0}\right], 0<\beta<1$ and $\eta_{k}$ are constants.

The problem (4.1)-(4.3) describes a model of a fractional problem in real world phenomena in which often some parameters are involved. The values of these parameters can be measured only up to certain degree of accuracy. Hence, the orders of fractional differential equation $\alpha$ in (4.1) and the initial conditions $\alpha-k$ in (4.2) may be subject to some errors either by necessity or for convenience. Thus, it is important to know how the solution of (4.1)-(4.3) changes when the values of $\alpha$ and $\alpha-k$ are slightly altered.

Theorem 4.1 Let $\alpha>0$ and $\delta>0$ such that $0 \leq n-1<\alpha-\delta<\alpha \leq n$. Also let $f: I \times \mathbb{R} \times$ $\mathbb{R} \rightarrow \mathbb{R}$ be a continuous function satisfying the assumption:

(A $\left.\mathrm{A}_{6}\right)$ There exist constants $L_{1}, L_{2}>0$ such that $\left|f\left(t, u_{1}, u_{2}\right)-f\left(t, v_{1}, v_{2}\right)\right| \leq L_{1}\left|u_{1}-v_{1}\right|+L_{2} \mid u_{2}-$ $v_{2} \mid$, for each $t \in I$ and $u_{1}, u_{2}, v_{1}, v_{2} \in \mathbb{R}$.

If $y$ and $z$ are the solutions of the initial value problems (4.1)-(4.3) and

$$
\begin{aligned}
& { }_{\mathrm{H}} D^{\alpha-\delta} z(t)=f\left(t, z(t), \max _{s \in[\beta t, t]} z(s)\right), \quad t \in I, \\
& \left.{ }_{\mathrm{H}} D^{\alpha-\delta-k} z(t)\right|_{t=t_{0}^{+}}=\bar{\eta}_{k}, \quad k=1,2, \ldots, n, n=-[-(\alpha-\delta)],
\end{aligned}
$$

with initial function

$$
z(t)=\bar{\phi}(t), \quad t \in\left[\beta t_{0}, t_{0}\right]
$$

respectively, where $\bar{\eta}_{k}$ are constants and $\bar{\phi}$ is a given continuous function on $\left[\beta t_{0}, t_{0}\right]$ such that $\phi(t) \not \equiv \bar{\phi}(t)$ for all $t \in\left[\beta t_{0}, t_{0}\right]$, then the following estimates hold for $t_{0}<t \leq h<T$ :

(I) Suppose $\alpha-\delta>\frac{1}{2}$. Then for $t \in I$

$$
\begin{aligned}
|z(t)-y(t)| \leq & t\left\{c_{5} A^{2}(t)+h_{5}(t)\right. \\
& \left.\times \exp \left(\frac{3 \Gamma(2 \alpha-2 \delta-1)\left(L_{1}^{2}+L_{2}^{2}\right)\left(t-t_{0}\right)}{\Gamma^{2}(\alpha) t}\right)\right\}^{\frac{1}{2}} .
\end{aligned}
$$

(II) Suppose $0<\alpha-\delta \leq \frac{1}{2}$. Then for $t \in I$

$$
\begin{aligned}
|z(t)-y(t)| \leq & t\left\{c_{6} A^{b}(t)+h_{6}(t)\right. \\
& \left.\times \exp \left(\frac{\left(3 \Gamma\left((\alpha-\delta)^{2}\right)\right)^{\frac{1}{\alpha-\delta}\left(L_{1}^{b}+L_{2}^{b}\right)\left(t-t_{0}\right)}}{\Gamma^{b}(\alpha) t}\right)\right\}^{\frac{1}{b}},
\end{aligned}
$$

where

$$
\begin{aligned}
A(t)= & \left|\sum_{j=1}^{n} \frac{\bar{\eta}_{j}}{\Gamma(\alpha-\delta-j+1)}\left(\log \frac{t}{t_{0}}\right)^{\alpha-\delta-j}-\sum_{j=1}^{n} \frac{\eta_{j}}{\Gamma(\alpha-j+1)}\left(\log \frac{t}{t_{0}}\right)^{\alpha-j}\right| \\
& +\left|\left(\log \frac{t}{t_{0}}\right)^{\alpha-\delta}\left(\frac{1}{\Gamma(\alpha-\delta+1)}-\frac{1}{(\alpha-\delta) \Gamma(\alpha)}\right)\right|\|f\|
\end{aligned}
$$




$$
\begin{aligned}
& \quad+\left|\frac{1}{(\alpha-\delta) \Gamma(\alpha)}\left(\log \frac{t}{t_{0}}\right)^{\alpha-\delta}-\frac{1}{\Gamma(\alpha+1)}\left(\log \frac{t}{t_{0}}\right)^{\alpha}\right|\|f\|, \\
& \|f\|=\sup _{t_{0} \leq t \leq h}\left|f\left(t, y(t), \max _{s \in[\beta t, t]} y(s)\right)\right|, \\
& b=1+\frac{1}{\alpha-\delta}, \\
& c_{5}=\max \left\{3 t_{0}^{-2},\left(\beta t_{0}\right)^{-2}\right\}, \\
& c_{6}=\max \left\{3^{\frac{1}{\alpha-\delta}} t_{0}^{-b},\left(\beta t_{0}\right)^{-b}\right\}, \\
& h_{5}(t)=c_{5} \max _{s \in\left[\beta t_{0}, t_{0}\right]}|\bar{\phi}(s)-\phi(s)|^{2}+\frac{3 c_{5} \Gamma(2 \alpha-2 \delta-1)}{\Gamma^{2}(\alpha) t} \int_{t_{0}}^{t}\left(L_{1}^{2} A^{2}(s)+L_{2}^{2} \max _{\xi \in[\beta s, s]} m_{2}^{2}(\xi)\right) d s
\end{aligned}
$$

and

$$
\begin{aligned}
h_{6}(t)= & c_{6} \max _{s \in\left[\beta t_{0}, t_{0}\right]}|\bar{\phi}(s)-\phi(s)|^{b} \\
& +\frac{c_{6}\left(3 \Gamma\left((\alpha-\delta)^{2}\right)\right)^{\frac{1}{\alpha-\delta}}}{\Gamma^{b}(\alpha) t} \int_{t_{0}}^{t}\left(L_{1}^{b} A^{b}(s)+L_{2}^{b} \max _{\xi \in[\beta s, s]} m_{2}^{b}(\xi)\right) d s,
\end{aligned}
$$

with a continuous function $m_{2}(t)$ is defined by

$$
m_{2}(t)= \begin{cases}A(t), & t \in I, \\ |\bar{\phi}(t)-\phi(t)|, & t \in\left[\beta t_{0}, t_{0}\right] .\end{cases}
$$

Proof The solutions $y$ and $z$ of the initial value problems (4.1)-(4.3) and (4.4)-(4.6) satisfy the following equations:

$$
y(t)=\sum_{j=1}^{n} \frac{\eta_{j}}{\Gamma(\alpha-j+1)}\left(\log \frac{t}{t_{0}}\right)^{\alpha-j}+\frac{1}{\Gamma(\alpha)} \int_{t_{0}}^{t}\left(\log \frac{t}{s}\right)^{\alpha-1} f\left(s, y(s), \max _{\xi \in[\beta s, s]} y(\xi)\right) \frac{d s}{s}
$$

and

$$
\begin{aligned}
z(t)= & \sum_{j=1}^{n} \frac{\bar{\eta}_{j}}{\Gamma(\alpha-\delta-j+1)}\left(\log \frac{t}{t_{0}}\right)^{\alpha-\delta-j} \\
& +\frac{1}{\Gamma(\alpha-\delta)} \int_{t_{0}}^{t}\left(\log \frac{t}{s}\right)^{\alpha-\delta-1} f\left(s, z(s), \max _{\xi \in[\beta s, s]} z(\xi)\right) \frac{d s}{s},
\end{aligned}
$$

respectively. So using the assumption $\left(\mathrm{A}_{6}\right)$, it follows that

$$
\begin{aligned}
|z(t)-y(t)| \leq & \left|\sum_{j=1}^{n} \frac{\bar{\eta}_{j}}{\Gamma(\alpha-\delta-j+1)}\left(\log \frac{t}{t_{0}}\right)^{\alpha-\delta-j}-\sum_{j=1}^{n} \frac{\eta_{j}}{\Gamma(\alpha-j+1)}\left(\log \frac{t}{t_{0}}\right)^{\alpha-j}\right| \\
& +\mid \frac{1}{\Gamma(\alpha-\delta)} \int_{t_{0}}^{t}\left(\log \frac{t}{s}\right)^{\alpha-\delta-1} f\left(s, z(s), \max _{\xi \in[\beta s, s]} z(\xi)\right) \frac{d s}{s} \\
& -\frac{1}{\Gamma(\alpha)} \int_{t_{0}}^{t}\left(\log \frac{t}{s}\right)^{\alpha-\delta-1} f\left(s, z(s), \max _{\xi \in[\beta s, s]} z(\xi)\right) \frac{d s}{s} \mid \\
& +\mid \frac{1}{\Gamma(\alpha)} \int_{t_{0}}^{t}\left(\log \frac{t}{s}\right)^{\alpha-\delta-1} f\left(s, z(s), \max _{\xi \in[\beta s, s]} z(\xi)\right) \frac{d s}{s}
\end{aligned}
$$




$$
\begin{aligned}
& -\frac{1}{\Gamma(\alpha)} \int_{t_{0}}^{t}\left(\log \frac{t}{s}\right)^{\alpha-\delta-1} f\left(s, y(s), \max _{\xi \in[\beta s, s]} y(\xi)\right) \frac{d s}{s} \mid \\
+ & \mid \frac{1}{\Gamma(\alpha)} \int_{t_{0}}^{t}\left(\log \frac{t}{s}\right)^{\alpha-\delta-1} f\left(s, y(s), \max _{\xi \in[\beta s, s]} y(\xi)\right) \frac{d s}{s} \\
& -\frac{1}{\Gamma(\alpha)} \int_{t_{0}}^{t}\left(\log \frac{t}{s}\right)^{\alpha-1} f\left(s, y(s), \max _{\xi \in[\beta s, s]} y(\xi)\right) \frac{d s}{s} \mid \\
\leq & A(t)+\frac{1}{\Gamma(\alpha)} \int_{t_{0}}^{t}\left(\log \frac{t}{s}\right)^{\alpha-\delta-1} \\
& \times\left(L_{1}|z(s)-y(s)|+L_{2}\left|\max _{\xi \in[\beta s, s]} z(\xi)-\max _{\xi \in[\beta s, s]} y(\xi)\right|\right) \frac{d s}{s} \\
\leq & A(t)+\frac{1}{\Gamma(\alpha)} \int_{t_{0}}^{t}\left(\log \frac{t}{s}\right)^{\alpha-\delta-1} \\
& \times\left(L_{1}|z(s)-y(s)|+L_{2} \max _{\xi \in[\beta s, s]}|z(\xi)-y(\xi)|\right) \frac{d s}{s}, \quad t \in I,
\end{aligned}
$$

where $A(t)$ is defined by (4.9), and

$$
|z(t)-y(t)|=|\bar{\phi}(t)-\phi(t)|, \quad t \in\left[\beta t_{0}, t_{0}\right]
$$

Applying Theorem 3.2 yields the desired inequalities (4.7) and (4.8). This completes the proof.

In the following theorem, we give the upper bounds of solution of the Hadamard fractional differential equation with 'maxima' and initial conditions (4.1)-(4.3).

Theorem 4.2 Assume that:

( $\left.\mathrm{A}_{7}\right)$ There exist functions $\mu, v \in C\left(I, \mathbb{R}_{+}\right)$such that for $t \in I, u_{1}, u_{2} \in \mathbb{R}$,

$$
\left|f\left(t, u_{1}, u_{2}\right)\right| \leq \mu(t)\left|u_{1}\right|+v(t)\left|u_{2}\right|
$$

If $y$ is solution of the initial value problem (4.1)-(4.3) such that $\phi(t) \not \equiv$ for all $t \in\left[\beta t_{0}, t_{0}\right]$, then the following estimates hold:

(III) Suppose $\alpha>\frac{1}{2}$. Then for $t \in I$

$$
\begin{aligned}
|y(t)| \leq & t\left\{c_{3}\left(\sum_{j=1}^{n} \frac{\left|\eta_{j}\right|}{\Gamma(\alpha-j+1)}\left(\log \frac{t}{t_{0}}\right)^{\alpha-j}\right)^{2}\right. \\
& \left.+h_{7}(t) \exp \left(\frac{3 \Gamma(2 \alpha-1)}{\Gamma^{2}(\alpha) t} \int_{t_{0}}^{t}\left\{\mu^{2}(s)+v^{2}(s)\right\} d s\right)\right\}^{\frac{1}{2}} .
\end{aligned}
$$

(IV) Suppose $0<\alpha \leq \frac{1}{2}$. Then for $t \in I$

$$
\begin{aligned}
|y(t)| \leq & t\left\{\frac{c_{4}\left|\eta_{1}\right|^{b}}{\Gamma^{b}(\alpha)}\left(\log \frac{t}{t_{0}}\right)^{b(\alpha-1)}\right. \\
& \left.+h_{8}(t) \exp \left(\frac{\left(3 \Gamma\left(\alpha^{2}\right)\right)^{\frac{1}{\alpha}}}{\Gamma^{b}(\alpha) t} \int_{t_{0}}^{t}\left\{\mu^{2}(s)+v^{2}(s)\right\} d s\right)\right\}^{\frac{1}{b}},
\end{aligned}
$$


where $b, c_{3}, c_{4}$ are defined as in Theorem 3.2,

$$
\begin{aligned}
h_{7}(t)= & c_{3} \max _{s \in\left[\beta t_{0}, t_{0}\right]} \phi^{2}(s)+\frac{3 c_{3} \Gamma(2 \alpha-1)}{\Gamma^{2}(\alpha) t} \\
& \times \int_{t_{0}}^{t}\left\{\mu^{2}(s)\left(\sum_{j=1}^{n} \frac{\left|\eta_{j}\right|}{\Gamma(\alpha-j+1)}\left(\log \frac{s}{t_{0}}\right)^{\alpha-j}\right)^{2}+v^{2}(s) \max _{\xi \in[\beta s, s]} m_{3}^{2}(\xi)\right\} d s
\end{aligned}
$$

and

$$
\begin{aligned}
h_{8}(t)= & c_{4} \max _{s \in\left[\beta t_{0}, t_{0}\right]}|\phi(s)|^{b} \\
& +\frac{c_{4}\left(3 \Gamma\left(\alpha^{2}\right)\right)^{\frac{1}{\alpha}}}{\Gamma^{b}(\alpha) t} \int_{t_{0}}^{t}\left\{\frac{\left|\eta_{1}\right|^{b} \mu^{b}(s)}{\Gamma^{b}(\alpha)}\left(\log \frac{s}{t_{0}}\right)^{b(\alpha-1)}+\nu^{b}(s) \max _{\xi \in[\beta s, s]} m_{3}^{b}(\xi)\right\} d s,
\end{aligned}
$$

with a continuous function $m_{3}(t)$ defined by

$$
m_{3}(t)= \begin{cases}\sum_{j=1}^{n} \frac{\left|\eta_{j}\right|}{\Gamma(\alpha-j+1)}\left(\log \frac{t}{t_{0}}\right)^{\alpha-j}, & t \in I, \\ |\phi(t)|, & t \in\left[\beta t_{0}, t_{0}\right] .\end{cases}
$$

Proof The solution $y$ of the initial value problem (4.1)-(4.3) satisfies the following equations:

$$
\begin{aligned}
y(t)= & \sum_{j=1}^{n} \frac{\eta_{j}}{\Gamma(\alpha-j+1)}\left(\log \frac{t}{t_{0}}\right)^{\alpha-j} \\
& +\frac{1}{\Gamma(\alpha)} \int_{t_{0}}^{t}\left(\log \frac{t}{s}\right)^{\alpha-1} f\left(s, y(s), \max _{\xi \in[\beta s, s]} y(\xi)\right) \frac{d s}{s}, \quad t \in I, \\
y(t)= & \phi(t), \quad t \in\left[\beta t_{0}, t_{0}\right] .
\end{aligned}
$$

For $\alpha>0$, by using the assumption $\left(\mathrm{A}_{7}\right)$, it follows that

$$
\begin{aligned}
|y(t)| \leq & \sum_{j=1}^{n} \frac{\left|\eta_{j}\right|}{\Gamma(\alpha-j+1)}\left(\log \frac{t}{t_{0}}\right)^{\alpha-j} \\
& +\frac{1}{\Gamma(\alpha)} \int_{t_{0}}^{t}\left(\log \frac{t}{s}\right)^{\alpha-1}\left(\mu(s)|y(s)|+\nu(s) \max _{\xi \in[\beta s, s]}|y(\xi)|\right) \frac{d s}{s}, \quad t \in I, \\
|y(t)|= & |\phi(t)|, \quad t \in\left[\beta t_{0}, t_{0}\right] .
\end{aligned}
$$

Hence Theorem 3.2 yields the estimate of the inequalities (4.11) and (4.12). This completes the proof. 


\section{Author details}

${ }^{1}$ Nonlinear Dynamic Analysis Research Center, Department of Mathematics, Faculty of Applied Science, King Mongkut's University of Technology North Bangkok, Bangkok, 10800, Thailand. ${ }^{2}$ Department of Mathematics, University of loannina, loannina, 451 10, Greece.

\section{Authors' information}

Sotiris K Ntouyas is a member of Nonlinear Analysis and Applied Mathematics (NAAM) Research Group at King Abdulaziz University, Jeddah, Saudi Arabia.

\section{Acknowledgements}

The research of J Tariboon is supported by King Mongkut's University of Technology North Bangkok, Thailand.

\section{Received: 25 April 2014 Accepted: 30 September 2014 Published: 16 Oct 2014}

\section{References}

1. Pachpatte, BG: On some generalizations of Bellman's lemma. J. Math. Anal. Appl. 5, 141-150 (1995)

2. Pachpatte, BG: Inequalities for Differential and Integral Equations. Academic Press, New York (1998)

3. Pachpatte, BG: Explicit bounds on certain integral inequalities. J. Math. Anal. Appl. 267, $48-61$ (2002)

4. Pachpatte, BG: Integral and Finite Difference Inequalities and Applications. Elsevier, Netherlands (2006)

5. Lipovan, O: A retarded Gronwall-like inequality and its applications. J. Math. Anal. Appl. 252, 389-401 (2000)

6. Belarbi, S, Dahmani, Z: On some new fractional integral inequalities. J. Inequal. Pure Appl. Math. 10(3), Article 86 (2009)

7. Dahmani, Z: On Minkowski and Hermit-Hadamard integral inequalities via fractional integration. Ann. Funct. Anal. 1, 51-58 (2010)

8. Dahmani, Z: New inequalities in fractional integrals. Int. J. Nonlinear Sci. 9, $493-497$ (2010)

9. Dahmani, Z: The Riemann-Liouville operator to generate some new inequalities. Int. J. Nonlinear Sci. 12, 452-455 (2011)

10. Shao, J, Meng, F: Gronwall-Bellman type inequalities and their applications to fractional differential equations. Abstr. Appl. Anal. 2013, Article ID 217641 (2013)

11. Thiramanus, $P$, Tariboon, J, Ntouyas, SK: Henry-Gronwall integral inequalities with 'maxima' and their applications to fractional differential equations. Abstr. Appl. Anal. 2014, Article ID 276316 (2014)

12. Hadamard, J: Essai sur l'étude des fonctions données par leur développement de Taylor. J. Math. Pures Appl. 8, 101-186 (1892)

13. Kilbas, AA, Srivastava, HM, Trujillo, JJ: Theory and Applications of Fractional Differential Equations. North-Holland Mathematics Studies, vol. 204. Elsevier, Amsterdam (2006)

14. Butzer, PL, Kilbas, AA, Trujillo, JJ: Compositions of Hadamard-type fractional integration operators and the semigroup property. J. Math. Anal. Appl. 269, 387-400 (2002)

15. Butzer, PL, Kilbas, AA, Trujillo, JJ: Fractional calculus in the Mellin setting and Hadamard-type fractional integrals. J. Math. Anal. Appl. 269, 1-27 (2002)

16. Butzer, PL, Kilbas, AA, Trujillo, JJ: Mellin transform analysis and integration by parts for Hadamard-type fractional integrals. J. Math. Anal. Appl. 270, 1-15 (2002)

17. Kilbas, AA: Hadamard-type fractional calculus. J. Korean Math. Soc. 38, 1191-1204 (2001)

18. Kilbas, AA, Trujillo, JJ: Hadamard-type integrals as G-transforms. Integral Transforms Spec. Funct. 14, 413-427 (2003)

19. Chinchane, VL, Pachpatte, DB: A note on some integral inequalities via Hadamard integral. J. Fract. Calc. Appl. 4, 1-5 (2013)

20. Chinchane, VL, Pachpatte, DB: On some integral inequalities using Hadamard fractional integral. Malaya J. Mat. 1, 62-66 (2012)

21. Sroysang, B: A study on Hadamard fractional integral. Int. J. Math. Anal. 7, 1903-1906 (2013)

22. Sudsutad, W, Ntouyas, SK, Tariboon, J: Fractional integral inequalities via Hadamard's fractional integral. Abstr. Appl. Anal. 2014, Article ID 563096 (2014)

23. Kilbas, AA, Srivastava, HM, Trujillo, JJ: Theory and Applications of Fractional Differential Equations. North-Holland Mathematics Studies, vol. 204. Elsevier Amsterdam (2006)

24. Bainov, D, Hristova, S: Differential Equations with Maxima. Pure and Applied Mathematics. Chapman \& Hall/CRC, New York (2011)

25. Hristova, S, Stefanova, K: Some integral inequalities with maximum of the unknown functions. Adv. Dyn. Syst. Appl. 6 , $57-69(2011)$

26. Tariboon, J, Thiramanus, P, Ntouyas, SK: Dynamic integral inequalities on time scales with 'maxima'. J. Inequal. Appl. 2013, 564 (2013)

27. Kuczma, M: An Introduction to the Theory of Functional Equations and Inequalities: Cauchy's Equation and Jensen's Inequality. Birkhäuser, Basel (2009)

10.1186/1029-242X-2014-398

Cite this article as: Thiramanus et al.: Integral inequalities with 'maxima' and their applications to Hadamard type

fractional differential equations. Journal of Inequalities and Applications 2014, 2014:398 